La

Révolution

française

\section{La Révolution française}

Cahiers de l'Institut d'histoire de la Révolution française

19 | 2021

Appropriation de la Révolution française dans

I'historiographie japonaise

\title{
Un historien japonais Inoue Kôji (1910-89) : ses travaux sur l'histoire moderne et contemporaine de France et l'Affaire de Chichibu à l'ère Meiji
}

Takashi Koï

\section{OpenEdition}

\section{Journals}

Édition électronique

URL : http://journals.openedition.org/lrf/4455

DOI : $10.4000 /$ Irf.4455

ISSN : 2105-2557

Éditeur

IHMC - Institut d'histoire moderne et contemporaine (UMR 8066)

Référence électronique

Takashi Koï, « Un historien japonais Inoue Kôji (1910-89) : ses travaux sur l'histoire moderne et contemporaine de France et l'Affaire de Chichibu à l'ère Meiji », La Révolution française [En ligne], 19| 2021, mis en ligne le 01 février 2021, consulté le 05 février 2021. URL : http:// journals.openedition.org/lrf/4455; DOI : https://doi.org/10.4000/Irf.4455

Ce document a été généré automatiquement le 5 février 2021

(C) La Révolution française 
Un historien japonais Inoue Kôji (1910-89) : ses travaux sur l'histoire moderne et contemporaine de France et l'Affaire de Chichibu à l'ère Meiji

Takashi Koï

\section{NOTE DE L'ÉDITEUR}

Meiji est le nom d'une ère du Japon allant du 8 septembre 1868 jusqu'au 29 juillet 1912 (à la mort de l'empereur Mutsuhito).

Nous avons conservé l'usage commun en Asie orientale des noms propres : le nom de famille en premier, le prénom en second. 


\section{Avant-propos}

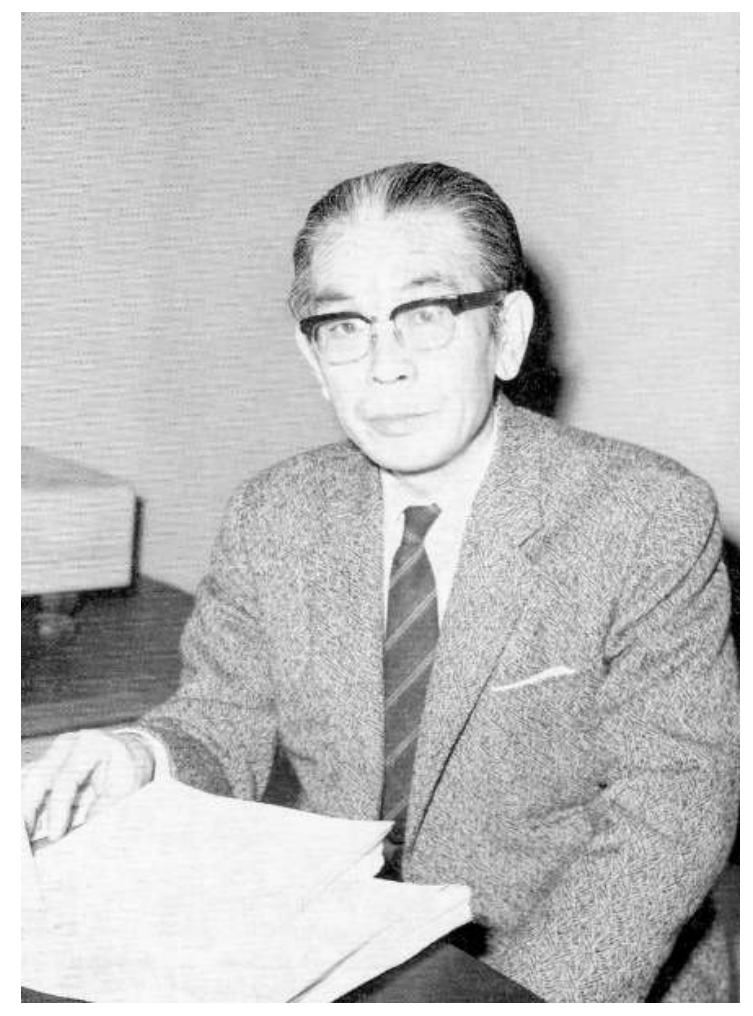

1 L'historien japonais Inoue Kôji joua un rôle important au Japon dans les études sur l'histoire moderne et contemporaine de la France et sur l'Affaire de Chichibu. Il est né en 1910 à Chichibu (dans le département de Saitama), qui se situe à environ soixantedix kilomètres au nord-ouest de Tokyo (à un peu moins de deux heures en train). Inoue termina ses études à l'Université impériale de Tokyo en 1935, puis fut nommé professeur d'histoire occidentale à l'Université de Kobe en 1953 après la parution de ses travaux pour le Grand dictionnaire de l'histoire du monde ${ }^{1}$.

Il publia d'autres travaux chez le libraire-éditeur Heibonsha pendant plusieurs années. Il fut ensuite professeur à Tokyo, d'abord à l'université de Rikkyô (Saint-Paul), de 1960 à 1975, puis à l'université de Tsuda-juku, de 1976 à 1986. Il a également séjourné en France pour ses études pendant une année en 1968-1969. Il a alors noué, avec le professeur Pierre Vilar (spécialiste de l'histoire sociale et économique de l'Espagne moderne), des relations d'amitié. Bien que l'on ne connaisse pas ses travaux sur l'histoire de France, car il n'a séjourné qu'une fois la France et n'a pas publié ses œuvres ou conférences en français, il est au Japon l'un des chercheurs les plus importants sur ce sujet, historien d'une génération insérée entre celle de Takahashi Kôhachirô (1912-82) et celle à laquelle appartiennent Shibata Michio (1926-2011), Chizuka Tadami (1932-2010) et Ninomiya Hiroyuki (1932-2006). Ses travaux ont ainsi beaucoup influencé les études de Chizuka et Ninomiya ${ }^{2}$.

3 Ses thèmes de recherches sont nombreux et divers, et peuvent être divisés en sept catégories comme suit :

1. Les Philosophes du $18^{\mathrm{e}}$ siècle en France, surtout Montesquieu et Rousseau.

2. Les problématiques relatives à la transition de la société féodale à la société moderne en Europe. 
3. La Révolution française, notamment au travers de Mirabeau et Robespierre.

4. Napoléon Bonaparte.

5. L'Histoire du $19^{\mathrm{e}}$ siècle, de la Restauration à la Commune de Paris en 1871.

6. L'École des Annales et l'historiographie en Europe.

7. L'Affaire de Chichibu en 1884.

\section{Traductions et commentaires en japonais des œuvres de Montesquieu et de Rousseau}

4 Inoue a été chargé en 1972 de rédiger le volume 28 de la collection «Ouvrages célèbres du monde", consacré à Montesquieu, et a traduit lui-même en japonais les Considérations sur les causes de la grandeur des Romains et de leur décadence ${ }^{3}$. De plus, en introduction de ce volume, il a rédigé une notice intitulée «La formation de la philosophie de Montesquieu au cours de sa vie ». Dans ce travail de soixante-huit pages, qui est considéré comme une étude authentique et originale sur Montesquieu, Inoue apporte des détails sur l'histoire de la famille de Montesquieu et sur la gestion de sa seigneurie. Il met en lumière les trois étapes de la formation de sa pensée. Les Lettres persanes, en 1721 , constituent une première étape pour passer de l'homme de lettres à l'historien, puis à l'homme réfléchissant sur la société. Montesquieu critique la culture de son époque et saisit la réalité de la société sous le règne de Louis XV. Pour L'historien japonais, on trouve une forte inspiration littéraire de moraliste dans ses Lettres persanes. Cependant, il ne saisit pas encore la réalité sociale comme objet d'analyse scientifique. Cette œuvre constitue une étude satirique concernant la société et la politique de son temps. En revanche Les Considérations sur les causes... présentent une description ordonnée de l'histoire de Rome. Montesquieu y recherche la loi de causalité dominante en histoire, suivant le processus historique de l'évolution de Rome en se fondant sur les documents qu'il rassemble. Finalement, Montesquieu, selon Inoue, devient fondateur de la «science positive » avec De l'esprit de lois (sa première édition a été publiée en 1876 au Japon). Cette œuvre a pour but d'étudier les règles scientifiques $\mathrm{du}$ fonctionnement des lois. Il érige le principe et en donne la preuve par la méthode déductive et positive. Quant au point de vue sur la séparation des pouvoirs, il faudrait, selon Inoue, nuancer l'apport de Montesquieu. Le concept de séparation des pouvoirs a été diversement interprété et utilisé par les savants en sciences politiques et les hommes politiques jusqu'à aujourd'hui. Le sujet essentiel de De l'Esprit de lois est la lutte contre le despotisme et l'arbitraire de l'Ancien Régime. L'œuvre offre une arme aux Parlements pour lutter contre la monarchie absolue. L'auteur y exprime sa pensée critique et son intention d'entourer le pouvoir exécutif, relevant du monarque, par le pouvoir judiciaire des Parlements et par le pouvoir législatif de la noblesse (l'assemblée de noblesse), capables de neutraliser la monarchie absolue et de mettre en place une monarchie modérée. Le point de vue de la séparation des pouvoirs est donc un système de défense pour justifier sa position sociale en tant que membre de la noblesse de robe, désirant instaurer une monarchie modérée. L'évolution de sa philosophie, que l'on peut trouver dans les Lettres persanes, les Considérations sur les causes (...) et De l'Esprit de lois, donne un exemple de la transformation de la reconnaissance sensible et littéraire en système scientifique. Cette évolution illustre comment l'intuition, née de l'observation sociale de la vie quotidienne, se transforme en concept scientifique, selon le professeur japonais. 
Quant à Rousseau, Inoue traduit en japonais $\mathrm{Du}$ Contrat social en 1966 (la première édition japonaise de cette œuvre a été publiée en 1882 par Nakae Chômin: son manuscrit traduit en japonais et ses copies à la main étaient lus parmi ceux qui se trouvaient autour de lui, dès 1874, au début du mouvement pour la liberté et les droits civiques). D'après le commentaire rédigé par Inoue, Rousseau a imaginé l'état civil (l'état social composé par les citoyens) et a réfléchi sur les systèmes législatifs des nations. Mais cette œuvre de théorie pure ne fut presque pas lue ni comprise. Ce qui était moins connu en Occident l'était encore davantage au Japon et devait être rappelé. Ce sont plutôt ses œuvres littéraires comme La Nouvelle Héloïse ou Émile qui font de Rousseau l'un des grands maîtres du $18^{\mathrm{e}}$ siècle au Japon. Ces œuvres, dans lesquelles il décrit concrètement ses idées sociales, ses passions de moraliste et son aspiration vers l'homme naturel, sont accueillies avec transport par de nombreux lecteurs.

Selon Inoue, la signification révolutionnaire du Contrat social apparut plus tard, lors de la Révolution française, après le moment où Marat a commenté cette œuvre, en public ou dans L'Ami du peuple. Inoue vulgarise également au Japon le débat ayant agité la Troisième République concernant la relation entre les idées de Rousseau et leur mise en pratique dans la Révolution française, d'après une observation d'Auguste Comte selon laquelle existaient deux filiations pour fonder la République sur un socle d'idées: de Diderot à Danton et de Rousseau à Robespierre, la première l'emportant dans les cercles officiels de la République institutionnelle ${ }^{4}$.

\section{Les problèmes relatifs à la transition de la société féodale à la société moderne en Europe}

7 Le second mérite de l'étude historique d'Inoue Kôji est la remise en cause de l'opinion généralement admise (celle de l'école Kôza ${ }^{5}$ et de l'école d'ôtsuka Hisao ${ }^{6}$ ) sur la formation des capitaux industriels (manufacture ou grande fabrique) au temps de la transition de la société féodale à la société moderne. L'école Kôza, dont faisait partie Takahashi Kôhachirô, auteur de La structure de la Révolution bourgeoise en 1950, considère que le capitalisme industriel est formé par la transformation de paysans riches en capitalistes ruraux ou entrepreneurs de manufacture (manufacturiers capitalistes, c'est-à-dire le mythe du yeoman - paysan à son compte et indépendant -, idée issue de L'Éthique protestante et l'esprit du capitalisme de Max Weber, telle qu'elle fut comprise au Japon). Cette dynamique constituerait le moteur fondamental du développement moderne. Cette théorie propose la relation concurrentielle entre les capitalismes mercantile et industriel. Dans un second temps, les capitalistes industriels (manufacturiers) ne surgissent que par la différenciation au sein de la paysannerie entre paysans riches et pauvres. Cette dernière doctrine est réfutée en France (selon Inoue Kôji, par exemple, G. Lefebvre ne relève pas de petits paysans-entrepreneurs qui dirigent des manufactures dans le département du Nord) et manque de preuves matérielles. Au contraire, Inoue estime que les artisans et négociants riches deviennent marchands-fabricants (les patrons-industriels de la grande fabrique) en vertu d'un processus de différenciation des maîtres de la communauté de métier ou des négociants-entrepreneurs qui se transforment en patrons-industriels de la grande fabrique. Ils finissent par dominer les petits producteurs paysans à la campagne pour augmenter la quantité de production au fur et à mesure de l'élargissement des marchés intérieurs et internationaux. Inoue est d'accord pour appeler ce système la 
manufacture séparée ou dispersée avec le putting-out system. Inoue explique cela dans un article, "L'industrie textile dans le Département du Nord », dans l'ouvrage Formation de l'industrie manufacturière en Europe moderne (p. 149-223) 7 . Le marchand-fabricant n'est pas seulement un marchand qui domine la circulation des marchandises, mais aussi un entrepreneur-manufacturier qui emploie des travailleurs domestiques dispersés. Ainsi, la transformation de maîtres riches appartenant à la communauté de métier en entrepreneurs-négociants marque précisément le début du capitalisme industriel de la société moderne. On peut dire que le triomphe de cet uklad capitaliste est lié au temps de la prospérité économique durant le second tiers du $18^{\mathrm{e}}$ siècle. Pourtant, selon Marx, le triomphe politique de la bourgeoisie industrielle ne se produit qu'au milieu de $19^{\mathrm{e}}$ siècle (lors de la Révolution de Février), à l'approche de la Révolution industrielle ${ }^{8}$. Encore une fois, ces discussions, qui pourraient sembler basiques en Europe après la seconde guerre mondiale, sont fort importantes dans un Japon qui s'ouvre au second temps de sa prospérité par l'abolition de tout signe de féodalité et la réforme agraire.

\section{La Révolution française et Napoléon Bonaparte}

8 Les liens entre la Révolution française et Napoléon Bonaparte sont également l'un des objets des recherches d'Inoue. Ce lien est moins prisé de nos jours en Europe, et surtout en France, dans la pratique historique, mais il était reçu comme une évidence dans le Japon de l'après-guerre, ce que n'avait point renié Georges Lefebvre, auteur de Napoléon en 1941. Depuis son article intitulé "Tendance actuelle des études sur la Révolution française, Aulard, Jaurès, Mathiez et Lefebvre " et publié en $1935^{9}$, Inoue ne cesse d'assimiler les résultats des études de la Révolution française en France et de remettre en question la société moderne créée par cette Révolution. Ainsi, il met en place une nouvelle image de la Révolution française fondée sur les résultats des études positives en France, et critique par là-même l'interprétation de la Révolution française de l'école Kôza. Ainsi, d'après elle, la révolution paysanne est accomplie durant la Révolution française. Les jacobins, qui représentent politiquement les intérêts de la petite bourgeoisie, abattent par la Terreur les girondins, qui sont les porte-parole du capital de l'époque préindustrielle (vors intflutlich-- Max Weber). Il en résultera que la Révolution française assure le développement des petits producteurs. L'école Kôza affirme donc que la Révolution française accomplit le programme de lutte paysanne et populaire. Contre cette illusion du triomphe de la révolution paysanne, Inoue affirme que l'on doit d'abord évaluer l'ampleur de l'anéantissement de la propriété (foncière) féodale. Par cette rupture, le paysan communautaire et assujetti à la seigneurie se transforme en paysan de la société moderne ${ }^{10}$.

Deuxièmement, la Révolution française ne nationalise pas les propriétés foncières des privilégiés et bourgeois, mais les biens ecclésiastiques et ceux des émigrés. Ainsi, la plupart des adjudicataires de biens nationaux sont des bourgeois citadins et des paysans aisés. La crise agraire (le manque de terre des paysans pauvres) sous l'Ancien Régime n'est pas donc dénouée par la vente des biens nationaux pendant la Révolution française. Autrement dit, la vente des biens nationaux ne change que peu la composition sociale de la paysannerie. Inoue nie ainsi le point de vue de l'école Kôza en vertu duquel la lutte paysanne parviendrait à sa fin (ce qui aurait rendu les paysans indépendants [petits propriétaires] et différents de tous les paysans indigents manquant de terre lors de la vente des biens nationaux) grâce à la Révolution française. 
10 De plus, il reconsidère les rôles de la Montagne, des enragés et des sans-culottes dans la Révolution française, à partir des études sur les mouvements paysans et sans-culottes selon Lefebvre, Soboul et Rudé. Contrairement au point de vue de l'école Kôza, les girondins et les montagnards y représentent les uns comme les autres les intérêts de la bourgeoisie, malgré des différences subtiles entre eux. Il n'y a pas de grande différence dans leurs idées politiques et économiques. On peut tenir pour une alliance calculée ce que Robespierre concède aux sans-culottes, prenant soin par ailleurs des intérêts de la bourgeoisie pour sauvegarder les conquêtes de la Révolution de $1789^{11}$. Quant à la relation entre jacobins et sans-culottes, contrairement à l'école Kôza qui les considère tous comme des petits bourgeois, selon lui, les jacobins représentent les intérêts de la bourgeoisie moyenne et ils ont des caractéristiques différentes politiquement et socialement des sans-culottes. Comme les historiens français le montrent, Robespierre réprime les enragés et réprime le mouvement sans-culotte dans la dernière phase de la Terreur, quoiqu'il continue la lutte pour fonder la République, en accord avec les sansculottes, contre les girondins jusqu'à la prise de contrôle de la Convention. Non qu'il existe une différence entre les girondins et les jacobins, car ceux-ci représentent le capital de la petite industrie et ceux-là le capital de l'époque préindustrielle, mais la différence se situe dans leurs politiques révolutionnaires. Les jacobins acceptent les exigences de la masse populaire en termes de reconnaissance citoyenne, voire se coalisent avec la masse populaire et forment le front patriotique opposé à la ContreRévolution intérieure et extérieure pour sauvegarder la République naissante. En revanche, les girondins prennent une attitude conciliante envers les forces modérées, pourvu que l'intérêt bourgeois remporte la victoire. N'est-ce pas l'essence des luttes de la Révolution française entre 1792 et 1793 ?

11 Pourtant, Inoue Kôji croit que la tâche essentielle imposée à la Révolution française est accomplie par la Déclaration des droits de l'homme et du citoyen de 1789 et la Constitution de 1791. La Constitution de 1795 est dans le prolongement de celle de 1791. S'intéressant au temps long et aux évolutions profondes de la société, Inoue constate que la fondation civile de la société inventée par la Révolution française est conservée également sous le régime impérial et le gouvernement réactionnaire de la Restauration ${ }^{12}$.

12 Pourtant la question des luttes sociales le retient aussi dans le temps court. Inoue souligne l'importance du rôle des enragés et des hébertistes dans le mouvement sansculotte. Ainsi, en plus des efforts de Robespierre pour rallier la masse sans-culotte aux jacobins (Robespierre partage des idées rousseauistes avec eux), on ne peut pas négliger le rôle des enragés et des hébertistes au moment de construire l'union des sans-culottes avec les robespierristes. Ils possèdent une dynamique et inventent des slogans fondés sur les aspirations des sans-culottes dans les sections, anticipant les exigences de ces derniers. On peut dire qu'ils sont le centre nerveux du mouvement sans-culotte.

13 Inoue remarque donc le rôle des "meneurs » comme les enragés dans son étude sur l'Affaire de Chichibu (la révolte paysanne dans le mouvement pour la liberté et les droits civiques à l'époque Meiji). C'est ici qu'il faut dépasser l'impression d'une lecture qui relèverait de la vulgate révolutionnaire pour comprendre son utilisation afin d'appréhender les dynamiques historiques et conflictuelles proprement japonaises et l'utilisation possible de catégories qui peuvent nous sembler schématiques mais utiles pour comprendre les luttes sociales dans le Japon de la fin du $19^{e}$ siècle. En outre, Inoue touche à la méthode analytique pour saisir les caractéristiques de mouvements 
populaires comme ceux des sans-culottes ou des paysans de Chichibu. Il souligne la nécessité de n'examiner point seulement le fondement social et la composition socioprofessionnelle des mouvements populaires, mais aussi les motifs revendiqués et subjectifs de leurs actions (conscience, aspirations et leur relation avec un système d'idées comme celui de Rousseau). Il affirme que l'on doit tenir compte de deux facteurs mentionnés ci-dessus pour comprendre la forme, l'origine, le but et la signification historique du mouvement populaire. Il critique la tendance déterministe (ou réductionniste) économique dans l'historiographie japonaise jusqu'en 1960-1970. On peut trouver dans cette remarque l'influence des études sur les mouvements populaires dans la Révolution française comme celles de Lefebvre. Pour Inoue Kôji, il s'est agi durant toute sa carrière d'étudier les motifs précis des révoltés et leurs pratiques ${ }^{13}$.

Enfin, ce sont les biographies de trois grandes personnages qu'il rédige sur l'histoire de la France révolutionnaire et napoléonienne qui le font connaitre des universitaires japonais. Il publie Mirabeau et la Révolution française (273 p.) en 1949, Napoléon (198 p.) en 1957 et Robespierre - Les mains sanglantes de Rousseau (284 p.) en $1962^{14}$. Ses réflexions sur les problèmes centraux aux différentes époques de la Révolution française et de l'Empire sont exprimées dans ces trois ouvrages. Il cherche à faire représenter les phases du début de la Révolution jusqu'à la fin de l'Empire par trois types de protagonistes (il est également le traducteur japonais du Napoléon d'Henri Calvet dans la collection "Que sais-je? $»^{15}$ ). Il a soin de les décrire, non seulement dans leur dimension privée, mais aussi en tant que personnages sociaux pris dans l'histoire. Ce faisant, il se reconnait dans la méthode mise en œuvre dans le Napoléon de Lefebvre, qui s'impose tel un modèle au Japon.

Inoue commente ainsi leurs personnalités: Robespierre n'est pas simplement un homme politique, mais il incarnerait le cerveau politique et la conscience de la Révolution; Danton, lui, regarde, raconte et utilise la réalité en tant que telle; tandis que Mirabeau est un homme politique réaliste chez qui la théorie dépend de la pratique, et accorde de plus une importance à l'action et à la manipulation des hommes dans la réalité politique, davantage qu'aux formules spéculatives que l'on prête volontiers, et sûrement de façon commode et réductrice, à Robespierre. Dans l'ensemble de ces travaux, on mesure mieux l'impact de Georges Lefebvre sur les spécialistes de la Révolution française au Japon après 1945. Le respect du maître en Sorbonne, sa science de la révolution paysanne dans un pays qui connait une mutation agraire sans précédent, sa façon de penser le socle des acquis civils finalement conservés par Napoléon, Empereur-- ce dernier mot ne pouvait qu'avoir un fort écho au Japon -, expliquent l'aura du professeur français sur tant de japonais.

\section{La France au $19^{\mathrm{e}}$ siècle}

Les études sur la France au $19^{\mathrm{e}}$ siècle étaient rares au Japon jusqu'à la première moitié des années 1960. En effet, les chercheurs japonais s'occupaient surtout des problèmes relatifs à la transition de la société féodale, à la société moderne en Europe, qui ne pouvaient qu'interpeller la génération des historiens japonais qui avaient vécu quinze ans auparavant la transformation totale des structures de propriété lors de la réforme agraire ayant suivi la Seconde Guerre mondiale. La transition de la société féodale et la force du paradigme marxiste employé pour l'étudier n'était pas un débat abstrait ou idéologique sur l'archipel, mais bien la réalité sociale de l'après-Seconde Guerre 
mondiale. De plus, les historiens japonais avaient également tendance à comprendre $l^{\prime}$ histoire de France au $19^{\text {e }}$ siècle au travers des écrits de Marx ${ }^{16}$. Dans pareille situation, $l^{\prime}$ Histoire du siècle des bourgeois (19e siècle) de Inoue est sans doute la première œuvre sur la France de cette période écrite par un historien japonais ${ }^{17}$. Elle devient un point de départ des monographies concernant divers sujets de l'histoire du $19^{\mathrm{e}}$ siècle, quoiqu'elle soit une histoire générale de France et des autres pays européens du temps de la Restauration jusqu'à la Commune de Paris. Inoue y affirme qu'il prête attention à la réciprocité entre culture politique et économie dans l'histoire européenne du $19^{\mathrm{e}}$ siècle. Il traduit aussi en japonais les Révolutions de 1848, ouvrage de Gaston-Martin publié dans la collection « Que sais-je ? »

Pourtant, son article le plus remarquable parmi ses travaux concernant la France de cette époque est «La signification actuelle de la Commune de Paris - Le concept de dictature prolétarienne chez Marx et la réalité de l'insurrection parisienne de $1871^{19}$ ». Il s'agit dans cette étude d'émettre un doute sur l'idée habituellement acceptée que la Commune de Paris aurait établi le premier gouvernement prolétarien dans l'histoire du monde. C'est cette vue que l'on retrouve dans le livre d'Histoire du Monde utilisé par les lycées de cette époque au Japon ${ }^{20}$.

Dans son article, Inoue cite trois textes de Marx - le «Deuxième appel » du Conseil général de la Première Internationale rédigé du 6 au 9 mai 1871 pendant l'Insurrection de Paris ; «La Guerre civile en France » du 30 mai 1871; et la Lettre au représentant (Domela Nieuwenhuis) du mouvement ouvrier hollandais du février 1882 (dix ans après la Commune de Paris) - pour vérifier les changements de ses interprétations sur la Commune de Paris et confronter les vues de Marx avec la réalité des faits historiques. Dans son « Deuxième appel ", selon Inoue, Marx penserait que les travailleurs parisiens ne satisfaisaient pas encore à la possibilité d'abattre le gouvernement républicain. Leur insurrection contre le pouvoir gouvernemental aurait été une conduite contreproductive, car il leur aurait fallu réaliser préalablement l'organisation d'un parti. Cependant dans "La Guerre civile en France ", Marx admire l'héroïsme des communards et proclame que la forme d'État établie par la Commune de Paris est le gouvernement de la classe prolétaire, c'est-à-dire le gouvernement de la dictature prolétarienne. Or, dans sa lettre au représentant hollandais Demela Nieuwenhuis, datée de février 1882, il écrit la Commune de Paris sous les traits d'une simple révolte urbaine suscitée dans des conditions extraordinaires sous l'occupation de l'armée prussienne. Les socialistes ne sont pas la majorité des membres de l'Assemblée sous la Commune de Paris. Il y aurait eu des avantages à faire des concessions au gouvernement versaillais plutôt que d'en venir à l'insurrection des masses populaires. Inoue Kôji remarque ce changement de point de vue sur la Commune de Paris. D'après Inoue, chez Marx, le point de vue sur les réalités sociales évolue en spirale, suivant que la considération sur l'histoire, la théorie et la politique pratique s'influencent réciproquement. Il en résulte que son point de vue sur la Commune de Paris change deux fois dans ses écrits de 1871 à 1882. Quel avis Inoue a-t-il alors sur la réalité de la Commune de Paris? Premièrement, il n'accepte pas la proposition selon laquelle la Commune de Paris établit le premier gouvernement prolétaire dans l'histoire du monde, car la majorité des travailleurs parisiens et les cadres de l'Internationale ne sont pas composés de travailleurs de grandes industries, mais de ceux de petits ateliers d'artisanat. Dans cette mesure, les prolétaires ne représentant que vingt-cinq des quatre-vingts membres du 
Conseil de la Commune : on ne peut donc pas affirmer que la Commune de Paris soit le gouvernement des travailleurs.

Deuxièmement, selon Inoue, après l'établissement du gouvernement municipal à Paris, la Commune de Paris a dû réformer la société civile pour réaliser l'émancipation sociale des travailleurs. Si l'on se demande comment la Commune de Paris a essayé de changer fondamentalement la structure économique et sociale de la société civile après le Second Empire, on est obligé de constater que la réforme n'a pu aboutir. Inoue en conclut que la Commune de Paris n'inspire pas aux contemporains de l'intérêt par son résultat, mais par sa leçon et sa postéritée ${ }^{1}$.

Inoue aborde ainsi les sujets divers de l'histoire moderne et contemporaine de la France, mais il ne manque pas non plus de porter un grand intérêt au développement remarquable des études d'histoire en France en son temps, c'est-à-dire aux problématiques et méthodes analytiques de la « Nouvelle Histoire».

\section{Assimilation de l'École des Annales}

Inoue Kôji apprécie l'École des Annales et assimile bien les études de ses fondateurs Marc Bloch et Lucien Febvre, Labrousse, Braudel et Le Roy Ladurie. Il traduit donc en japonais les œuvres suivantes pour les faire connaitre au Japon : La société française, 1789-1960 de George Dupeux, Fête et Révolte d'Yves-Marie Bercé, Recueil d'articles de Fernand Braudel et Montaillou de Le Roy Ladurie ${ }^{22}$. Dans les commentaires de ses traductions, il revient sur les origines et les tendances novatrices de l'École des Annales avec les carrières et travaux des auteurs. Là encore, il faut comprendre son rôle de passeur, de vecteur de la culture historique française au Japon. D'autre part, dans son article «L'origine des études de l'École des Annales » en 1979, il éclaircit ce que Henri Berr avance, avant l'École des Annales, soit « la Synthèse historique au lieu de l'histoire événementielle ou l'histoire historisante ${ }^{23} »$. Cette conception de la Synthèse historique correspond, ajoute-t-il, à sa conception selon laquelle l'histoire est la science synthétisant la réalité sociale et historique. Quant à Marc Bloch et Lucien Febvre, Inoue apprécie bien ce qu'ils soulignent sur l'importance de l'actualité historique et la nécessité de l'histoire totale. Ils ont pour objet d'études, l'histoire de toutes les phases de la vie sociale des hommes et des groupes humains, en introduisant les méthodes analytiques des sciences voisines avec l'histoire (non seulement l'économie et la géographie, mais la sociologie, l'ethnologie, l'anthropologie, la psychologie collective, la météorologie et les autres sciences sociales).

De plus, l'École des Annales est à l'origine de la méthode d'histoire quantitative et sérielle, avec Simiand et Labrousse. Cela est, dit-il, devenu une révolution de la méthode analytique pour l'École des Annales. Il publie donc l'édition japonaise de $L a$ Société française, 1789-1960 de Dupeux en 1968. Il remarque que le dessein de l'histoire sociale, chez Labrousse, comporte le schéma de "conjoncture économique - groupes sociaux - aspects de conscience des hommes ${ }^{24} »$. Dès lors, il accepte et adopte ce schéma.

En ce qui concerne la deuxième génération des Annales, Inoue mentionne Braudel, qui façonne les concepts de la longue durée, du Monde méditerranéen, et cherche à mettre en place une histoire totale. En développant les échanges scientifiques avec LéviStrauss, Althusser, Sartre et les autres chercheurs des sciences voisines de l'histoire, il influence les successeurs de l'École des Annales. De plus, Inoue apprécie que les 
chercheurs de la deuxième génération entrent dans le subconscient, les mentalités, les sociabilités (pour ainsi dire, le « software ${ }^{25}$ pour mieux comprendre les hommes dans la réalité historique). Pour faire connaître leurs résultats aux lecteurs japonais, il publie les éditions japonaises des œuvres de Bercé et de Le Roy Ladurie. À l'occasion des éditions japonaises des œuvres de Bercé et de Le Roy Ladurie, il découvre, dans la première, une méthode d'analyse de la révolte paysanne et, dans la seconde, une superbe ethnologie historique, qu'il accueille chaleureusement, fondée sur l'étude minutieuse du monde rural tout comme son étude sur les Paysans du Languedoc.

Inoue n'accueille pas toujours toutes les études des Annales sans critique. Les chercheurs d'histoire occidentale au Japon étaient définitivement influencés par Marx et Weber jusqu'aux années 1970. Il ne cesse donc de mettre en question la correspondance entre les concepts de la Nouvelle Histoire et ceux du marxisme. Pour lui, il s'agit de vérifier la compatibilité ou l'incompatibilité entre l'histoire des structures économiques et sociales chez Marx et l'histoire quantitative et sérielle des conjonctures économiques et sociales des Annales. Il mentionne Pierre Vilar (l'auteur de La Catalogne dans l'Espagne moderne) comme un des rares historiens qui tient compte de ce sujet ${ }^{26}$.

Les trois points suivants montrent et résument son attitude à l'égard de l'étude de l'histoire.

Premièrement, il est un historien qui ne cesse de se demander comment l'étude de l'histoire contient la possibilité de comprendre les circonstances actuelles de la société. L'histoire recoupe le concept d'utilitarisme en ce qu'elle incarne une forme scientifique d'étude du passé pour faire comprendre le présent. Par conséquent, une attention particulière doit être portée aux méthodes du métier d'historien ${ }^{27}$.

Deuxièmement il signale le manque d'études positives sur l'histoire occidentale au Japon jusqu'à son époque et souligne l'importance de ce chantier, citant souvent une expression d'un historien : avoir "l'aile de feu et les souliers en plomb ${ }^{28}$ ». Selon lui, «l'aile de feu » signifie la théorie, "les souliers en plomb» les sources, l'analyse des sources et le processus de synthèse des faits. Il dit que ces ailes et souliers sont imposés à l'étude d'histoire. Il cite Les paysans du Nord pendant la Révolution de Lefebvre comme le meilleur exemple d'étude d'histoire suivant ce schéma.

Troisièmement il répond à la question des rapports entre l'histoire et la fiction. Selon lui, la première est fondée sur des connaissances établies, depuis qu'elle s'est formée comme science en Europe il y a deux siècles. Certaines sciences sociales - comme l'histoire, la science des lois et l'économie politique - élaborent leurs théories par le maniement des données du passé. En somme, les sciences sociales en viennent à élaborer des systèmes théoriques d'après les faits historiques. Selon Inoue, l'histoire est une science fondamentale, en ce sens qu'elle fournit les données du passé sur lesquelles les sciences sociales élaborent les systèmes théoriques. L'histoire devient la science synthétisante des éléments divers (sociaux, politiques, culturels, religieux, idéologiques, de la vie quotidienne etc.) de la société humaine, différence radicale avec la fiction et l'imaginaire ${ }^{29}$. Inoue assimila donc complètement les études d'histoire sur la France moderne et contemporaine avec les travaux de l'École des Annales et les présenta aux chercheurs 
japonais en histoire, ainsi qu'aux lecteurs japonais. Lui-même a tenté de les appliquer à son étude sur l'Affaire de Chichibu, une grande insurrection de paysans dans son pays natal à la fin de $19^{e}$ siècle. Il a constaté, en conséquence, que les méthodes analytiques de l'étude de l'histoire en France s'avéraient fort utiles aux études de l'histoire des pays étrangers comme le Japon. De plus, il a pu démontrer certaines relations directes et indirectes entre une insurrection de paysans japonais et l'histoire moderne française.

\section{L'Affaire de Chichibu}

Inoue Kôji est le premier chercheur à s'être occupé de l'Affaire de Chichibu, en même temps qu'il continuait son travail sur l'histoire de la France moderne. Il est très rare au Japon qu'un spécialiste de l'histoire occidentale fasse également des recherches sur des sujets liés à l'histoire de son pays. «Les sans-culottes parisiens et le Parti des Indigents [paysans endettés] de Chichibu [Chichibu konmintô] sont », disait-il, « mes jumeaux ${ }^{30}$ ».

31 Le district de Chichibu est une région rurale et montagneuse qui produit de la soie grège. La soie représente 60 à $70 \%$ des exportations du Japon pendant les années 10 de Meiji (1878-87). De plus, 80 à $90 \%$ du contingent d'exportation de la soie est représenté par la soie grège. C'est en 1858 (dix ans avant le début de Meiji) que la soie grège du Japon est exportée pour la première fois en France. Jusqu'à 1884, l'année de la révolte de Chichibu, la France était le pays importateur le plus important de la soie grège venant du Japon. Elle représentait en moyenne $44 \%$ des exportations japonaises de soie grège de 1873 à 1877, majoritairement pour le marché lyonnais.

Chichibu est situé au cœur de la région qui produisait la soie. De fait, depuis l'ouverture du pays aux étrangers à la fin de l'époque d'Edo ${ }^{31}$, la plupart des paysans de Chichibu ne vivaient plus que du produit de la soie grège (élevage des vers à soie et filage) et l'exploitation agricole n'était pour eux qu'une ressource d'appoint. Par exemple en 1888, $96 \%$ des familles paysannes pratiquaient l'élevage des vers à soie. Pourtant la soie grège est un produit spéculatif sur le marché international. Son prix fluctue considérablement selon la quantité de récolte de cocons et la conjoncture économique en Europe. Les paysans pratiquant l'élevage des vers à soie et le filage devinrent aisés durant les années de hausse des prix (comme en 1870, 1875 et 1880). Au contraire, le prix de la soie baissa considérablement avec la récession économique après 1881 , se dépréciant notamment de $50 \%$ pendant les trois années de 1881 à 1884 .

Lyon avait également été frappée par la Grande Dépression de 1876 à 1886. En outre, la production dans la Grande Fabrique de Lyon en 1884 s'effondra encore de $50 \%$ par rapport à celle de l'année précédente, déjà en pleine crise économique. Les paysans de Chichibu qui produisaient la soie grège furent touchés de plein fouet par la dépression mondiale. La politique déflationniste adoptée par le ministre des Finances Matsukata Masayoshi, la pénurie d'argent et l'augmentation des impôts locaux aggravèrent encore la situation. Par conséquent, les paysans qui tombaient dans l'indigence furent obligés d'emprunter de l'argent aux usuriers. Suivant l'usage de ce temps-là, 10 yens de dette (en fait, une recette nette de 8 yens à cause des prélèvements de $20 \%$ ) donnaient 26 yens et 16 sens onze mois plus tard. Autre exemple, 100 yens de dette donnaient 220 yens (avec un intérêt de 120 yens) un an après. Les paysans qui faisaient faillite perdaient maison et propriété. Ceux qui prenaient la fuite et disparaissaient étaient nombreux. Tous ces paysans appauvris formèrent la base de ce Parti des Indigents. Leur collectivité pressait les usuriers d'accepter le délai d'acquittement et les versements 
échelonnés au moyen de la négociation collective. Ce mouvement qui admonestait les usuriers retient l'attention d'Inoue. L'une des caractéristiques de l'Affaire de Chichibu réside aussi dans le fait que plusieurs membre du Parti de la Liberté ainsi que plusieurs membres du Parti libéral (Jiyu-tô) ${ }^{32}$ participèrent à l'Insurrection du Parti des Indigents de Chichibu.

On peut donc dire que l'Affaire constitue un prolongement du mouvement pour la liberté et les droits civiques (Jiyû-Minken undô). Le Parti de la Liberté, qui était un parti d'opposition, fut formé par Itagaki Taisuke ${ }^{33}$ en 1881 avec pour objectif l'établissement d'une Constitution et l'ouverture d'un Parlement. Ôi Kentarô ${ }^{34}$ était le chef de la gauche de ce parti et responsable permanent pour les cinq départements de la région du Kantô ${ }^{35}$. Il visita Chichibu en février 1884, neuf mois avant l'Insurrection des paysans endettés, et y donna des conférences publiques. À cette occasion, une vingtaine de petits ou moyens paysans adhérèrent au Parti de la Liberté. Quelques mois plus tard, en mai, on compte une trentaine de membres. Parmi eux, on trouve plusieurs meneurs et militants radicaux actifs du Parti des Indigents de Chichibu, qui avait comme président Tashiro, un paysan moyen déchu, producteur de soie grège, joueur de dés professionnel et arbitre entre usuriers et paysans endettés. Pourtant, on doit noter qu'ils n'établissaient pas toujours des relations étroites avec les dirigeants du Parti de la Liberté. L'Insurrection n'est pas provoquée par les directives de celui-ci; d'ailleurs, pour la stopper, le Parti de la Liberté envoya un commissaire extraordinaire à Chichibu.

$\mathrm{Au}$ mois d'août, dans un contexte de prix bas de la soie grège, les militants actifs du Parti des Indigents mobilisèrent les paysans endettés pour des réunions en forêt (en raison de l'existence d'une habitude du jeu d'argent en forêt parmi les paysans de Chichibu) afin de constituer leur réseau en mouvement secret. Un groupe de dirigeants se constitua. Il dressa la liste de dettes des paysans de plus de trente villages affiliés au Parti des Indigents (le district de Chichibu se compose de quatre-vingt-quatre communes) et il requit un délai d'acquittement auprès du commissariat de police, en même temps qu'il entamait des négociations individuelles avec les usuriers. Ses requêtes furent pourtant repoussées par la police et les usuriers, parmi lesquels Inoue note, dans les archives, la présence d'un "vieil avare " que l'historien compare à une sorte de père Goriot nippon. Le 26 octobre 1884, le groupe de meneurs fixa le début de l'insurrection pour le $1^{\mathrm{er}}$ novembre, repoussant la demande d'ajournement du soulèvement formulée par le Parti de la Liberté. L'Insurrection commença en fait dans les villages de l'est de Chichibu dès le 31 octobre. Trois mille insurgés occupèrent la cité d'Ômiya (la principale ville de Chichibu) le 2 novembre. Ce jour-là, les insurgés étaient sept à dix mille, soit environ un sixième de la population du district. Le Parti des Indigents plaça en conséquence toute la région de Chichibu sous son influence. C'est " la Commune de Chichibu», explique Inoue Kôji. Le Parti des Indigents exigait des usuriers et des autorités sur place, les points suivants : un délai de dix ans pour le remboursement des dettes et des versements échelonnés sur quarante ans; la fermeture de l'école pour exonération d'impôts locaux ${ }^{36}$; l'allégement de l'impôt sur des revenus divers; et l'allégement de la charge fiscale sur le village. Les insurgés brûlèrent les reconnaissances de dettes, démolirent ou incendièrent les maisons des usuriers qui n'acceptaient pas les exigences du Parti des Indigents. Ils imposèrent également un emprunt forcé aux marchands riches. Ils rejetèrent les Okuin-chô (registres de contrat de vente des biens immobiliers avec les sceaux de responsable de 
village) conservés dans les mairies de village. Ils assaillirent les commissariats de police et tirèrent sur les policiers et gendarmes.

Cependant, du 3 au 4 novembre, tous les points de fuite du pays plat furent fermés par la gendarmerie. Le groupe des meneurs perdit alors tout espoir d'un soulèvement général des paysans dans tous les départements de la région du Kantô. Les meneurs principaux quittèrent le siège du Parti des Indigents dans l'après-midi du 4 et le quartier général de l'Insurrection fut démantelé le soir même.

Pourtant, un aspect important de l'Affaire de Chichibu réside dans ce que l'Insurrection ne se termina pas avec la fuite de ses dirigeants. D'une part, un détachement de l'armée insurrectionnelle, composé de cinq cents insurgés, continua à lutter. Il livra combat contre la garnison gouvernementale dans le district de Kodama. Il fut écrasé par cette dernière et dix insurgés furent tués au combat. D'autre part, un autre détachement de cent vingt insurgés, dirigé par Kikuchi Kanpei (chef d'état-major de l'insurrection) et Sakamoto Shûsaku (porteur d'ordre, l'un des meneurs radicaux), franchit le col de Jukkoku et envahit le département de Nagano, au nord-ouest de Chichibu, pour étendre l'Insurrection. Cependant, il fut attaqué par des policiers et gendarmes à Managashi (ancien nom de la cité de Kômi, dans le département de Nagano) le 9 novembre. Les insurgés s'enfuirent, en déroute, après treize tués au combat. C'était la fin de l'Affaire de Chichibu.

Dès le début de son analyse, Inoue souligne la nécessité de tenir compte des aspects sociaux, mais aussi des aspects politiques pour saisir les caractéristiques de ce mouvement populaire. Ainsi, il cherche à analyser la vie rurale de paysans de Chichibu, la composition sociale, les âges, la conscience collective, les taux d'analphabétisme et de condamnation antérieure, l'organisation du Parti des Indigents, les idées et les rôles de meneurs, la relation entre le Parti des Indigents de Chichibu et le Parti de la Liberté. Il est clair qu'Inoue applique les méthodes analytiques de l'étude sur le mouvement populaire (sans-culotte ou rural) en France à son travail sur l'Affaire de Chichibu. Voyons à quels résultats mène son étude sur l'Affaire de Chichibu. Inoue met en valeur la phase économique défavorable et la dépression de la Grande Fabrique à Lyon en arrière-plan de l'Affaire de Chichibu, y rattachant directement la dette et l'indigence des paysans qui vivent du produit de la soie grège .

Deuxièment l'Insurrection est différente des révoltes paysannes de l'époque précédente (Edo) par son importance, sa dynamique et son organisation. Selon Inoue, cela est dû à ce que l'Affaire de Chichibu se produit dans le prolongement du mouvement pour la liberté et les droits civiques. Il examine donc le rapport entre les dirigeants et les meneurs du Parti des Indigents et la gauche du Parti libéral (surtout Ôi Kentarô), l'organisation du Parti des Indigents, et l'influence de la pensée moderne sur les objectifs de l'Insurrection.

41 Ôi Kentarô, le chef de la gauche du Parti libéral et le responsable permanent pour les cinq départements de la région du Kantô où se trouve le district de Chichibu, lit le français et est devenu un spécialiste de la science politique et des lois françaises. Il connaît également les idées de Rousseau et il écrit La loi agraire (Tochi heibun hô) plusieurs années après l'Affaire de Chichibu. Il propose l'acquisition par l'État de terrains cultivés appartenant aux grands propriétaires et leur partage à raison d'un 
chôbu (un hectare) pour chaque paysan pauvre. Plusieurs dirigeants et meneurs du Parti des Indigents adhèrent au Parti de la Liberté au moment de sa visite à Chichibu. Ils accueillent avec sympathie la promesse solennelle du Parti de la Liberté telle qu'exprimée dans les statuts: "Notre parti doit tenter de développer la liberté, sauvegarder les droits (civiques), accroître le bonheur et améliorer la société.» Inoue affirme que les meneurs paysans adhérèrent aux idées révolutionnaires du Parti de la Liberté. Pourtant, ce dernier, comme cela a été évoqué, a essayé d'arrêter l'Insurrection, perçue comme imprudente, par l'intermédiaire de Ôi Kentarô. En outre, le Parti de la Liberté prononce sa dissolution le 29 octobre 1884 (deux jours avant l'Insurrection du Parti des Indigents).

On trouve aussi plusieurs dirigeants et meneurs du Parti des Indigents qui connaissent bien les mouvements de pensée en France et dans les autres pays européens. Par exemple, d'après Inoue, Ide Tamekichi (receveur de fonds pour les dépenses militaires) est un bon connaisseur du monde rural à l'époque du mouvement pour la liberté et des droits civiques. Il a une collection de livres (en traduction) concernant la science politique et la législation en Occident, comme Le Code civil de Napoléon Bonaparte, $L a$ Révolution française de Mignet et Social Statics de Spencer. Tashiro Eisuke (président du Parti) justifie d'ailleurs l'Insurrection à la cour de justice en soutenant que l'usure est interdite par la loi en France.

Selon Inoue, le Parti des Indigents n'accueille pas seulement les slogans et points de vue du Parti de la Liberté, mais il les interprète à sa guise, grâce à la conscience politisée des paysans indigents. Le mouvement adopte des idées révolutionnaires comportant une signification sociale. Pour le Parti des Indigents, la liberté ne signifie pas seulement la liberté politique, mais l'émancipation de la misère dans laquelle sont tombés les paysans de Chichibu. Cela implique la libération du joug de l'usure, alors que le Parti de la Liberté ne remet pas en cause cette dernière. Inoue considère que le Parti des Indigents prône un égalitarisme social, distinct de l'égalitarisme des droits civiques revendiqué par le Parti libéral (dont les revendications de liberté politique passent par l'établissement de la Constitution et la convocation d'une Assemblée nationale).

Lorsque les paysans prétendent établir le "Yonaoshi » (action de corriger les maux et de sauver le monde), cela signifie la « réforme des affaires d'état» et le «nivelage du monde » pour améliorer les conditions de vie, Inoue Kôji écrit : «Je distingue le point de vue social sur la liberté et les droits civiques (Jiyu-Minken social) avec celui politique (Jiyu-Minken politique) dans les idées du mouvement pour la liberté et les droits civiques. Je pense que l'on peut faire avancer l'étude sur le mouvement pour la liberté et les droits civiques, en y apportant la conception de distinction entre les deux couches de la démocratie politique et de la démocratie sociale ${ }^{37}$. »

Troisièmement, Inoue voit les relations entre le Parti de la Liberté, les dirigeants et les paysans endettés du Parti des Indigents de Chichibu comme ressemblant à celles qu'entretiennent les jacobins avec les enragés et les sans-culottes : 


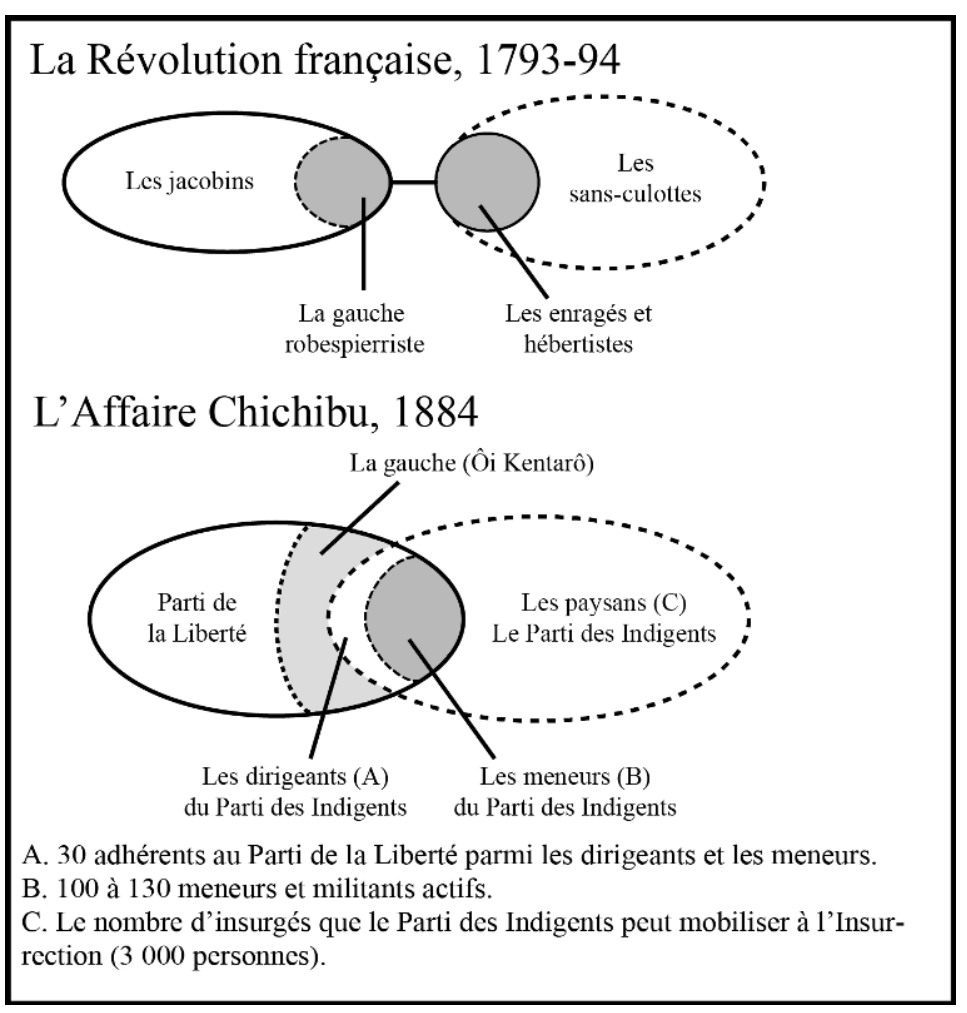

Des groupes réciproquement hétérogènes (les jacobins et les sans-culottes ) sont liés par les robespierristes dans la Révolution française. De même, dans l'Affaire de Chichibu, le Parti de la Liberté et le Parti des Indigents sont liés par les dirigeants du Parti des Indigents qui sont également membres du Parti de la Liberté. Ce sont les meneurs qui jouent le rôle le plus important car ils formulent les idées qui vont permettre au mouvement de déclencher l'Insurrection et de diriger les troupes insurgées. Ils correspondent aux enragés dans le mouvement sans-culotte. Les meneurs comme Sakamoto Shusuke (porteur d'ordres, condamné à mort à la cour), Takagishi Zenkichi (chef de section de village, condamné à mort) et Ochiai Toraichi (second chef de bataillon Otsu [X]), qui sont sous la direction du vice-président Katô Orihei (condamné à mort), apparaissent partout, mobilisent les paysans de village, assistent aux réunions en forêt et deviennent également représentants des pétitionnaires de village.

Inoue Kôji les nomme le «Trio du Parti des Indigents ». Selon lui, il existe une trentaine de meneurs dans le même genre. Un des militants actifs et radicaux, Ôno Naekichi (second chef de bataillon Kou $[\mathrm{Y}]$, mort à la guerre) force ainsi les paysans à participer à l'Insurrection: " Je me permets de vous dire. Aidez-nous, parce que nous allons nous opposer à Sa Majesté (l'Empereur Mutsuhito [Meiji]). Sinon nous vous tuerons à coups de sabre. "Ide Tamekichi (receveur du fonds pour dépenses militaires) porte sur les reçus l'inscription «la Direction révolutionnaire» ou «le Siège du Parti révolutionnaire » (il existe au moins trois reçus comme cela). Au dire de Arai Teikichi (paysan du département de Gunma ${ }^{38}$ ), Sakamoto Shusaku lui dit: «Si la chose va bien, on écrasera les usuriers et banques, on nivellera le monde et on sauvera le peuple. » Inoue perçoit un décalage relatif entre la conception des dirigeants (Tashiro Eisuke [président du Parti des Indigents] ou Inoue Denzô [son vice-président]) et celle des meneurs (Ide Tamekichi et Ôno Naekichi), similaire aux différences existant entre les robespierristes et les enragés. Les dirigeants, comme Tashiro ou Inoue Denzô, qui ont 
des conceptions politiques comparables à celles d'Ôi et de la gauche du Parti de la Liberté, font attention à la légalité politique et soutiennent l'ajournement juste avant l'Insurrection. Ce n'est que sous la poussée des militants actifs et radicaux qu'ils se décident à l'Insurrection et la provoquent. Lorsque les dirigeants comprennent l'impossibilité de l'insurrection générale dans tous les départements de la région du Kantô, ils quittent la direction de l'Insurrection le 4 novembre. Néanmoins, un détachement d'insurgés dirigé par Kikuchi Kanpei (chef d'état-major) et Sakamoto Shusuke (porteur d'ordre) continue l'Insurrection avec une volonté énergique jusqu'au 9 novembre comme évoqué ci-dessus.

Cette Insurrection a une organisation tout à fait différente des révoltes paysannes de ce temps. Voici la structure interne du Parti des Indigents lors de l'Insurrection

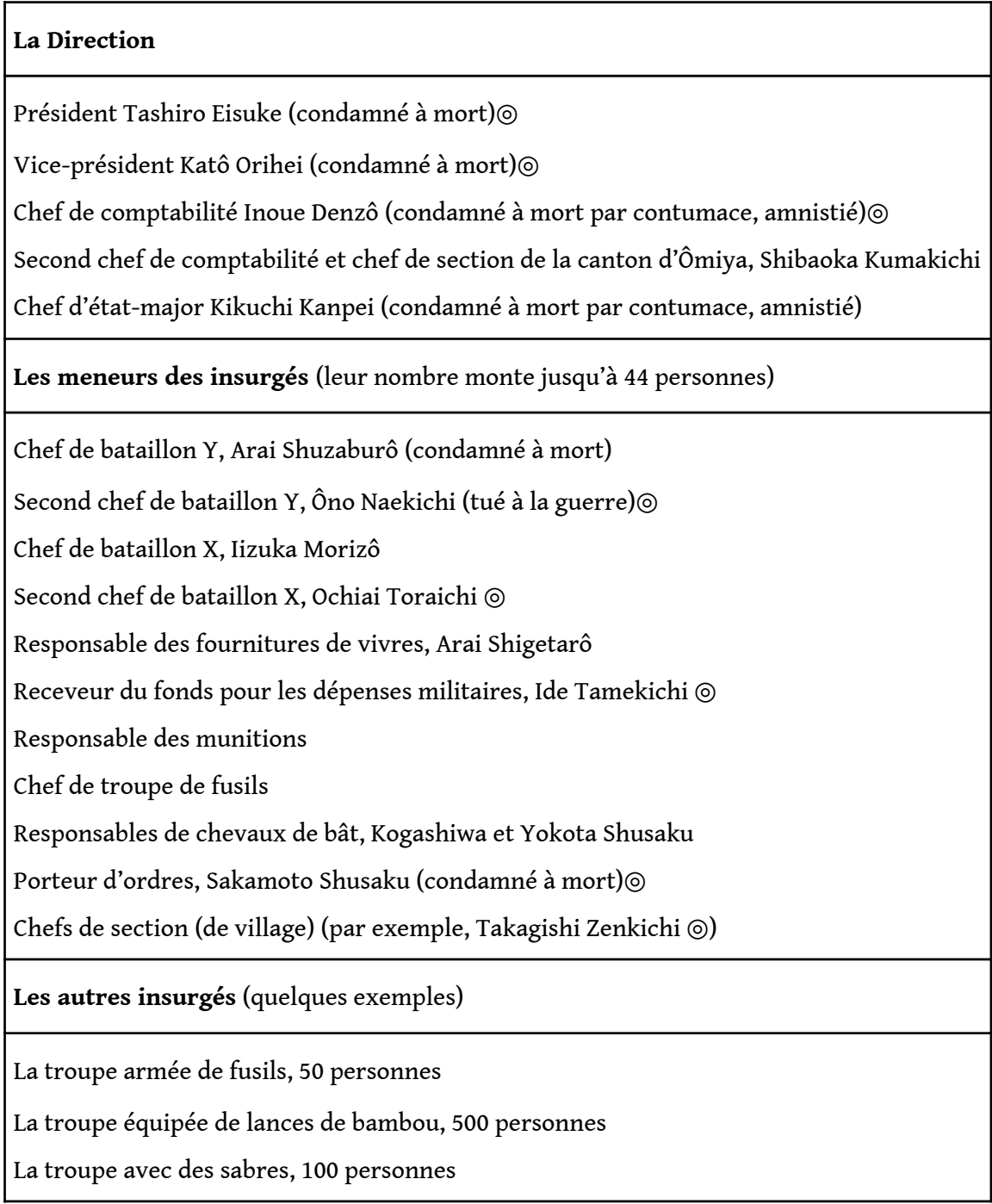

Le symbole @ indique l'adhésion au Parti de la Liberté.

De plus, la Direction donne à l'armée insurgée une discipline militaire composée de cinq articles comme : «Ceux qui pillent de l'argent ou des objets par intérêt seront tués à coups de sabre. » Que le Parti des Indigents possède une telle organisation militaire et livre, de fait, bataille aux policiers, gendarmes et troupes est exceptionnel parmi les soulèvements paysans à l'époque de Meiji. Inoue Kôji apprécie donc l'Affaire de 
Chichibu comme un moment insurrectionnel décisif dans le cadre du mouvement pour la liberté et les droits civiques.

Le Parti des Indigents est un collectif qui surgit dans le but de satisfaire aux exigences des paysans endettés. Il maitrise les motivations idéologiques de sa révolte, mobilise quelques milliers de paysans de Chichibu et se trouve capable d'organiser la lutte contre l'État pendant dix jours. Cet ensemble de faits a constitué une grande révolte sans exemple analogue depuis la guerre de Seinan (la guerre entre Satsuma et le gouvernement de Meiji ${ }^{39}$ ) en 1877. Il est également remarquable que les paysans de Chichibu avaient déjà une mentalité hostile aux fonctionnaires comme les policiers, gendarmes et garnisons. L'esprit d'indépendance, dix-sept ans après la Restauration de Meiji, avait perduré. Longtemps tu, cet événement est aussi au fondement de la démocratie au Japon moderne. Pour s'en rendre compte, il suffit d'étudier les mesures prises par le pouvoir judiciaire contre les insurgés après l'Insurrection. Bien que le commissariat de police départementale estime à six ou plus de sept mille le nombre total des insurgés (en fait, 4186 insurgés sont jugés au cour), les insurgés condamnés à mort sont sept dirigeants ou meneurs (dont deux dirigeants condamnés à mort par contumace, acquittés par amnistie lors de la publication de la Constitution de 1889 [année du centenaire de la Révolution française]) et quatre inculpés de meurtre de policier. D'autre part, 296 insurgés sont condamnés par la cour à une peine grave ${ }^{40}$.

Pour achever cet exposé, je voudrais relier l'Affaire de Chichibu et l'histoire de France. On peut d'abord mentionner deux points de relation directe entre les épisodes historiques.

Premièrement, les exigences du Parti des Indigents sont partiellement appuyées par la science politique et la philosophie de la France et des pays européens aux $18^{\mathrm{e}}$ et $19^{\text {e }}$ siècles, tout autant qu'elles proviennent des nécessités de la vie des paysans endettés. Au moins plusieurs dirigeants et meneurs connaissent bien les idées modernes des pays européens, preuve des origines partagées des deux événements, du moins du point de vue des idées.

Deuxièmement, on doit relier l'Affaire de Chichibu à la conjoncture économique internationale. Les petits producteurs de la soie grège à Chichibu tombent dans l'indigence à cause de la récession économique internationale, surtout en lien avec la Grande Dépression de la Fabrique de soie de Lyon. Ceux qui forment le Parti des Indigents sont des paysans de ce type.

Quant à la relation indirecte entre les deux, on peut relever également deux points. Tout d'abord, en ce qui concerne la relation entre le parti de la Liberté, ses factions et le mouvement populaire, il est possible de trouver une composition politique identique entre certaines époques de la Révolution française et le mouvement pour la liberté et les droits civiques. La relation entre les jacobins, les enragés et la masse sans-culotte correspond à celle qui existe à Chichibu entre la gauche du Parti de la Liberté et les dirigeants, les meneurs et les paysans insurgés du Parti des Indigents. On peut donc saisir la structure politique du mouvement pour la liberté et les droits civiques, en gardant à l'esprit l'étude sur le mouvement populaire sous la Révolution française. 
Par ailleurs, ainsi que les historiens de la Révolution française distinguent la démocratie sociale de la démocratie politique, Inoue adopte ce point de vue dans l'histoire de la Révolution française et l'étude sur le mouvement pour la liberté et les droits civiques au Japon afin de distinguer démocratie sociale et démocratie libérale.

Pour conclure, Inoue Kôji fut un historien unique qui a toujours œuvré à la compréhension des révolutions, capables par son érudition de lier des mouvements insurrectionnels aussi différents à plus de dix-sept mille kilomètres de distance. Les rébellions et les Révolutions française et japonaise étaient construites et pensées comme deux miroirs déformant mais renvoyant à des réalités pensables ensemble.

Comme pour mieux l'illustrer, l'historien avait deux parties dans le bureau de son appartement, l'une était réservée à l'histoire de France, l'autre à l'Affaire de Chichibu...

\section{NOTES}

1. Sekai rekishi dai jiten (Grand Dictionnaire de l'Histoire du Monde), 1952, Heibonsha.

2. De ce dernier, on lira en français Le Japon pré-moderne, 1573-1867, CNRS éditions, 2017.

3. Voir Montesukyu, sekai no meicho dai 28 kan (Montesquieu, les ouvrages célèbres du monde, vol.28), édition traduite en japonais par Inoue Kôji, 1972, Chuôkôronsha.

4. RUSO, Shakai-keiyaku-ron (ROUSSEAU, Du Contrat social), Édition traduite en japonais par Inoue Kôji , 1966, Chukô shinsho.

5. L'école Kôza est le nom que se donne un groupe de théoriciens marxistes regroupés autour de la rédaction d'un ouvrage collectif en sept volumes Nihon shihonshugi hattatsushi kôza, (Cours/Leçon sur l'histoire du développement du capitalisme japonais), publié en 1932-1933. Dans ce " cours », le régime Meiji marque le point de départ du Japon moderne et est analysé comme la mise en place d'un État absolutiste considéré comme «le stade suprême du féodalisme ». Cet ouvrage propose une analyse suffisamment percutante et originale de la société japonaise pour qu'il devienne le mot éponyme d'un courant de pensée en tant que tel : « leçons » ou « cours », en japonais kôza, a en effet donné naissance, en jargon de l'époque, au mot qui désigne ce courant marxiste d'historiens et d'économistes proches du parti communiste japonais, l'école Kôza (kôza ha). L'influence de ce courant sera déterminante dans toutes les années de l'après-guerre.

6. Ôtsuka Hisao (1907-1996) est un historien de l'économie, proche du marxisme, qui fut le premier à introduire au Japon l'oeuvre de Max Weber. Il créa autour de lui un courant de pensée original autour de la notion de modernité dans le Japon de la seconde moitié du $20^{\mathrm{e}}$ siècle.

7. INOUE Kôji (dir.), Yoroppa kindai-Kôgyô no seiritsu (La Formation de l'industrie manufacturière et moderne en Europe), 1961, Tôyô-keizai-shinposha.

8. Ibid.

9. Rekishigaku-kenkyû (Recherches historiques), 16 février 1935.

10. Voir INOUE Kôji, «Furansu-kakumei-hottan to tenbô » (La Révolution française - Origine et perspectives), Rekishi-hyôron, 21, 1950, et ID., «Furansu-kakumei-kenkyû no hansei-Ruso to 
Robesupieru wo megutte » (Réflexion sur les études relatives à la Révolution française au Japon autour de Rousseau et de Robespierre), Shien, 1968.

11. Inoue écrit ainsi : «Robespierre ne permet pas même un "fantôme de loi agraire". Selon ce dernier, l'égalité des biens est une chimère. Il conclut que l'égalité des biens est dans son principe irréalisable et le projet d'une communauté fondée sur l'égalité des biens n'est qu'une chimère. » Voir «Furansu-kakumei kenkyû no hansei-Ruso to Robesupieru o megutte ( Réflexion sur les études relatives à la Révolution française - Autour de Rousseau et Robespierre) », 1968, dans Kindaishi-zô no mosaku-Furansu-kakumei to Chichibu-jiken (Recueil d'articles: Études sur l'histoire moderne et contemporaine de la France et du Japon: la Révolution française et l'Affaire de Chichibu), Tokyo, Kashiwa shobô, 1976, p. 35 et 41. Voir INOUE Kôji, Robesupierru-Ruso no chinurareta te (Robespierre - Les mains sanglantes de Rousseau), Tokyo, Seibundô shinkôsha, 1962.

12. Voir INOUE Kôji, Robesupierru-Ruso no chinurareta te, op. cit.

13. Voir INOUE Kôji, Kindaishi-zô no mosaku..., op. cit.

14. Voir INOUE Kôji, Mirabo to Furansu-kakumei (Mirabeau et la Révolution française), 1949, Tokyo, Mokusuisha ; ID., Naporeon (Napoléon), 1957, Tokyo, Iwanami shinsho ; ID., Robesupierru-Ruso no chinurareta te, op. cit.

15. Il s'agit de la traduction de Henri CALVET, Napoléon, «Que sais-je?», parue en japonais, 1966, aux éditions Hakusuisha. Voir Naporeon densetu no seiritu (La formation de la légende napoléonienne), Shisô, 1970.

16. Voir INOUE Kôji, Marukusu ha gofu janai ([Les entretiens avec Eguchi Bokurô] Marx n'est pas un talisman [pour l'étude de l'histoire]), Tokyo,Yûzankaku shuppan, 1981.

17. INOUE Kôji, Burujowa no seiki (Histoire du siècle des bourgeois [19e siècle]), Sekai no rekishi, 12 (Histoire du monde, vol.12), Tokyo, Chuôkôronsha, 1961.

18. Traduction de GASTON-MARTIN, Les Révolutions de 1848, («Que sais-je?»), Tokyo, Hakusuisha, 1954.

19. INOUE Kôji, « Pari-Komyun no konnichiteki-igi-Marukusushugi-kokkaron no kaname o nasu puroretaria-dokusai to 1871 nen hôki no genjutsu ha dô kasanaruka ", Chuôkôron, n 1010, 1971. Cet article est repris dans INOUE Kôji, Kindaishi-zô no mosaku..., op. cit., p.65-89.

20. Ibid., p.72. Inoue ne cite pas l'exemple du manuel Histoire du monde utilisé dans les lycées en son temps. Pourtant cette remarque est pertinente au Japon.

21. Au Japon, certains chercheurs de l'histoire occidentale ou japonaise étaient socialistes ou communistes (on chantait l'Internationale à la fin du congrès annuel de la Rekishigaku kenkyûkai [Société savante d'étude d'histoire], l'une des plus grandes sociétés savantes d'histoire au Japon, jusqu'à la fin des années 1960). Ils participaient parfois au mouvement socialiste et révolutionnaire du Japon de l'après-guerre. Ils s'intéressaient donc à la Commune de Paris, qu'ils la tenaient comme le premier modèle de gouvernement prolétaire en se fondant sur « La Guerre civile en France » de Marx. Pourtant en 1971, l'année du centenaire de la Commune de Paris, Inoue a permis une meilleure compréhension de la réalité de la Commune et a remis en question sa signification historique.

22. Georges DUPEUX, La Société française, 1789 1960, édition en japonais traduite par Inoue K, Takemoto T, et alii, Tokyo, Tôyô keizai shinposha, 1968 ; Yves-Marie BERCÉ, Fête et Révolte. Des mentalités populaires du XVIème au XVIIIème siècle, Paris, Hachette, 1976 (coll. « Le Temps et les Hommes »), édition en japonais traduite par Inoue K., Matsudaira M. et Koï T., 1980 ; Recueil d'articles de Fernand Braudel, par. Inoue K. et traduite en japonais par Akashi M. et alii, Tokyo, Shinhyôron, 1989 ; Emmanuel LE ROY LADURIE, Montaillou, village occitan de 1294 à 1324, 2 vol., édition en japonais traduite par Inoue K et alii, Tokyo, Tôsui shobô, 1990-1991. Inoue s'est entretenu avec Le Roy Ladurie des méthodes analytiques d'histoire (à propos de son Montaillou) le 23 novembre1983 à Tokyo. Voir INOUE Kôji, Rekishi to wa nanika (Qu'est-ce qu' une histoire ?), Tokyo, Fujiwara shoten, 1991, p.238-279. 
23. INOUE Kôji, «Anaru-gakuha no seiritsu-kiban » (L'origine des études de l'École des Annales), Rekishi-hyôron, $\mathrm{n}^{\circ}$ 54, 1979.

24. Comme exemples de "conscience des hommes» (ishiki-keitai), Inoue mentionne les idéologies, les mentalités collectives, les consciences sociales, la qualité de la conscience religieuse, etc. INOUE Kôji, Rekishi wo kataru-gakusei to no taiwa (Parler de l'étude de l'histoireEntretiens avec les étudiants), Tokyo,Nigensha, 1979, p.129.

25. Inoue qualifie de software («logiciel») les sujets d'étude (par exemple, les éléments de la vie quotidienne) et leurs méthodes et instruments analytiques pour comprendre profondément la société des hommes, comme ceux de l'École des Annales (depuis sa deuxième génération ) mentionnés ci-dessus, par opposition au hardware (« équipement»), c'est-à-dire les catégories classiques utilisées dans l'étude de l'histoire comme les institutions politiques et sociales, la seigneurie, la monarchie absolue, la Révolution bourgeoise, le capitalisme industriel, la Révolution industrielle, le socialisme, etc.

26. INOUE Kôji, Supein ni okeru kakumei to kaikaku (La révolution et la réforme en Espagne), et ID., KindaiYoroppa rekishigaku [riron to hôhô] (Les études de l'histoire en Europe moderne [théories et méthodes]), Iwanami kôza sekai-rekishi (Cours d'Iwanami : Histoire du monde) $\mathrm{n}^{\circ} 30$, Tokyo, Iwanami shoten, 1971.

27. Voir INOUE Kôji, Kiki toshite no gendai-rekishi-gakusha no taiwa (Les entretiens avec Eguchi Bokurô - Regards des historiens sur les crises dans le monde contemporain), Tokyo, Sanseidô-shinsho, 1971.

28. Cette expression, qui pourrait provenir de Leopold von Ranke, est employée notamment dans INOUE Kôji (dir.), Yoroppa Kindai-kôgyô (La formation de l'industrie), p.152 ; et ID., Rekishi to wa nanika, op. cit., p.58.

29. INOUE Kôji, Rekishi wo kataru-gakusei to no taiwa (Parler de l'étude de l'histoire - Entretiens avec les étudiants), Tokyo, Nigensha, 1979, p.42.

30. INOUE Kôji, Kindaishi-zô noMosaku, op. cit., p.188.

31. Edo est l'ancien nom de Tokyo. L'époque d'Edo correspond à celle du shogounat des Tokugawa (1603-1868).

32. Le Parti de la Liberté est un des premiers partis politiques à l'échelle nationale au Japon; il joua un grand rôle dans le mouvement pour la liberté et les droits civiques (Jiyû-Minken undô). Il se forma en 1881 et fut dissous en 1884 (l'année de l'Affaire de Chichibu).

33. Itagaki Taisuke (1837-1919) était un ancien samouraï du fief de Tosa (département de Kôchi, dans la région de Shikoku). Après avoir participé à la Guerre de Boshin contre le shogounat des Tokugawa, il fut nommé conseiller du gouvernement de Meiji. Après sa démission, il fonda le Parti de la Liberté, dont il fut nommé président en 1881. Après 1896, il a occupé à plusieurs reprises le poste de ministre de l'Intérieur.

34. Ôi Kentarô (1843-1922) était l'un des meneurs du mouvement pour la liberté et les droits civiques. Fils d'un paysan du département d'Ôita à Kyûshû, il a fait des études occidentales à Nagasaki. Il participa à la Guerre de Boshin comme canonnier de l'armée gouvernementale (du shogounat). Après la Restauration de Meiji, il fut employé comme traducteur d'ouvrages politiques français au ministère de la Guerre. Après avoir quitté la fonction publique, il devint avocat, puis professeur de droit dans une école privée, et participa au Parti de la Liberté en 1881. Il devint l'un des meneurs les plus radicaux du mouvement pour la liberté et les droits civiques. En 1885, un après de l'Affaire de Chichibu, il est incarcéré pour complicité dans une tentative des anciens membres de la gauche du Parti de la Liberté au Japon de venir en aide au Parti indépendant de Corée (Affaire d'Ôsaka). Amnistié lors de la proclamation de la Constitution en 1889, il est élu membre de la Chambre des représentants en 1894.

35. La région du Kantô d'aujourd'hui est composée de Tokyo et des 6 départements limitrophes. 
36. INOUE Kôji, Chichibu jiken (L'Affaire de Chichibu), Tokyo, Chûkô-shinsyo,1968, p.51. Un impôt local permettait de financer l'administration et la maintenance des écoles primaires sur place. Les paysans endettés exigeaient la fermeture provisoire de l'école pour alléger cette charge, lourde pour eux.

37. INOUE Kôji, Kindaishi-zô no mosaku, op. cit., p.199.

38. Gumma est l'un des départements de la région de Kantô.

39. Satsuma est l'ancien nom du département de Kagoshima. Les samouraïs de Satsuma ont été l'une des forces principales de la guerre de Boshin contre le gouvernement d'Edo (shogounat) en 1868.

40. Voir INOUE Kôji, Chichibu-jiken shiryô shûsei (Recueil des sources relatives à l'Affaire de Chichibu), Tokyo, Nigensha, 6 vol., 1984-1989.

\section{RÉSUMÉS}

Inoue Kôji (1910-89) joua un rôle important dans les études sur l'histoire moderne de la France dans la seconde moitié du $20^{\mathrm{e}}$ siècle au Japon. Ses travaux peuvent être divisés en quatre catégories : les philosophes du $18^{\mathrm{e}}$ siècle en France, les problèmes sur la naissance de capitalisme industriel en France, les biographies de protagonistes de la Révolution française, et les autre sujets historiques.

Par ailleurs, Inoue est premier chercheur à s'être occupé de l'Affaire de Chichibu (1884) tout en menant en même temps son travail sur l'histoire de la France moderne. Chichibu était une région rurale qui produisait de la soie grège, et dont presque la moitié de la production est destinée à la France les années 1870 et 80 . L'une des qualités de l'Affaire de Chichibu réside dans ce que quelques dizaines de membres du Parti pour la liberté et les droits civiques participèrent à l'insurrection. Ils connaissaient bien les pensées modernes de la France. Inoue appliqua les méthodes analytiques de l'étude sur la Révolution française à son étude sur l'Affaire de Chichibu. En conséquence, il constata quatre points pour une relation directe et indirecte entre cette Affaire et l'histoire moderne de la France.

Inoue Kôji (1910-89) held a prominent position in the study of France's modern history during the second half of the $20^{\text {th }}$ century in Japan. His works can be divided in the following categories: the $18^{\text {th }}$-century's French philosophers, the issues about the birth of industrial capitalism in France, the biographies of the French Revolution's main actors, and the other historical topics. Inoue was the first researcher that studied the Chichibu Incident (1884) in addition to the history of modern France. Chichibu was a rural region that produced raw silk, and nearly half of its production was sold to France in the 1870s and 80s. One of the notable features of the Chichibu Incident was that several dozen members of the Freedom and People's Rights Movement played a role in the insurrection. They were very familiar with France's modern ideas. Inoue applied the analytical methods used for the study the French Revolution to that of the Chichibu Incident. Consequently, he concluded there were four direct and indirect connections between the Incident and France's modern history. 
INDEX

Mots-clés : Mouvement pour la liberté et les droits civiques, Jiyu-Minken Undo, Affaire de Chichibu (1884), Insurrection paysanne, Paysans endettés, Japon, Ère Meiji

Keywords : Freedom and People's Rights Movement, Jiyu-Minken Undo, Chichibu Incident (1884), Peasant uprising, Impoverished peasants, Japan, Meiji Period

\section{AUTEUR}

TAKASHI KOÏ

Rikkyou University 